\title{
The early gaseous and stellar mass assembly of Milky Way-type galaxy halos
}

\author{
Gerhard Hensler ${ }^{1,2}$ and Mykola Petrov ${ }^{1}$ \\ ${ }^{1}$ Dept. of Astrophysics, Univ. of Vienna, Tuerkenschanzstr. 17, 1180 Vienna, Austria \\ email: gerhard.hensler@univie.ac.at \\ ${ }^{2}$ Nat. Astron. Obs. of Japan, 2-21-1 Osawa, Mitaka-shi, Tokyo 181-8588, Japan
}

\begin{abstract}
How the Milky Way has accumulated its mass over the Hubble time, whether significant amounts of gas and stars were accreted from satellite galaxies, or whether the Milky Way has experienced an initial gas assembly and then evolved more-or-less in isolation is one of the burning questions in modern astronomy, because it has consequences for our understanding of galaxy formation in the cosmological context. Here we present the evolutionary model of a Milky Way-type satellite system zoomed into a cosmological large-scale simulation. Embedded into Dark Matter halos and allowing for baryonic processes these chemo-dynamical simulations aim at studying the gas and stellar loss from the satellites to feed the Milky Way halo and the stellar chemical abundances in the halo and the satellite galaxies.
\end{abstract}

Keywords. Galaxy: formation, Galaxy: halo, Galaxy: stellar content, galaxies: halos, galaxies: formation, galaxies: abundances

\section{Introduction}

In the past half century, by means of more sensitive observations of the Milky Way (MWG) stellar halo its formation was interpreted by two different processes: The first prefers the monolithic collapse (Eggen et al. 1962), and the second is the accretion model by Searl \& Zinn (1978). At the same time, White \& Rees (1978) proposed their Cold Dark Matter (CDM) hierarchical clustering paradigm in which galaxies are results from cooling and fragmentation of residual gas within the transient potential wells provided by the DM. In this framework galaxy formation proceeds in a "bottom up" manner starting with the formation of small clumps of gas inside DM subhalos, which then merge hierarchically into larger systems (Blumenthal et al. 1984, Springel et al. 2005).

CDM simulations of cosmological structure and galaxy formation predict the existence of a large number of such DM subhalos surrounding massive DM gravitational potentials. These subhalos should serve as the DM progenitors of dwarf galaxies (DGs) which indeed permeate the Local Group (LG), most of them concentrated as satellites around the MWG and M31. The closer to a mature galaxy they live, the more gas-free they are like elliptical DGs and are, therefore, called dwarf spheroidals (dSphs). Because of their low surface brightness, though even close to the MWG, for a long time only a few of them could be observed in the range of $\mathrm{M}_{V}=-14^{m}$ to $-10^{m}$ separated clearly from Globular Clusters. Their number increased over the last years thanks to systematic surveys like SDSS shifting the lower brightness limit by the recently discovered ultra-faint DGs (UFDs) to almost $-2^{m}$ (see e.g. Belokurov et al. 2010, Belokurov et al. 2014) so that they extend the DG sequence to its faintest end.

Theoretically already expected and verified by numerical simulations (Johnston et al. 2008), satellites in the neighbourhood and, thereby, in the tidal field of mature galaxies lose continuously gas and stars, the later observable as tidal streams (Lynden-Bell ${ }^{2}$ 1995, 
Jerjen et al. 2013). Due to their loss of orbital energy and angular momentum their fate is the partly disruption and their death as individuals is the accretion by their mature galaxy. This scenario of tidal disruption is at present most strikingly demonstrated by the Sagittarius DG (Ibata et al. 1994) with its tidal tails wrapped around the MWG (Majewski et al. 2003).

The CDM merging hypothesis requires the infall and accumulation of the MW mass not only by DM subhalos and gas but also by stars. If this "mining" of the halo with dSph stars (Salvadori et al. 2008) has happened in the very early epoch with the first stars only, no differences at the low Z end will tell us about as long as the MW halo stars are formed from the same gaseous substrate. At larger metallicities the $\alpha /$ Fe ratio of dSphs, however, declines already (due to supernova type Ia (SNIa) enrichment) while the halo stars are systematically at the constant value of SNII enrichment at the same [Fe/H] (Shetrone et al. 2003, Tolstoy et al. 2003, Venn et al. 2004, Koch et al. 2008; see also reviews by Koch (2009) and Tolstoy et al. (2009)). This fact allows to pin-down that progenitors of present-day dSphs are not the expected building blocks of the galactic halo and to explain the lack of the observed number of stellar streams. From kinematics of halo stars, however, a dichotomy is found by Carollo et al. (2007) and Bell et al. (2008), one regular population in the inner region of about $10 \mathrm{kpc}$ radius and an outermost heterogeneous and decoupled halo population most plausibly accreted from disrupted satellites. The metallicity distribution function (MDF) of the UFDs suggests that these tiny systems contain a larger fraction of extremely metal-poor stars than the MW halo does (Kirby et al. 2008) and witness the chemical imprint of the interstellar medium (ISM) when the Universe was less than 1 Gyr old.

Detections of hyper metal-poor stars (Beers \& Christlieb 2005) in the galactic halo and their peculiar element abundances (see e.g. Frebel et al. (2005)) opened a new field of galactic archeology, namely, modelling the element production by the first stars in the halo as well as in the UFDs towards understanding the zero metallicity nucleosynthesis and studying the formation of the halo. Chemical evidences for this scenario, especially in the metal-poor stellar content of the galactic halo, is mentioned also by Frebel et al. (2010), Frebel \& Bromm (2012), and others. On the other hand, it is currently unclear how the metal-poor MDF tail of the classical DGs, in which extremely metal-poor stars are absent, compares with that of the halo and the UFDs.

\section{Modeling the Milky Way satellite system}

In contrast to the DM evolution of subhalos treated by pure N-body simulations, the evolution of the baryonic component is much more complex because of the physical processes at work, such as star formation (SF), gas cooling, dissipation, energy and mass feedback. Baryonic gas loses kinetic energy dissipatively and thermal energy by radiation leading to cooling and gravitational collapse, while stellar radiation and winds as well as SNe lead to energy and chemical feedback. Almost all modelling up to now is dedicated to investigate the effects of various processes on the dSph evolution separately or the dSph evolution as an isolated system.

Although the gasdynamical simulations of dSphs advanced from $1 \mathrm{D}$ chemo-dynamical models by Hensler et al. (2004) to 3D (see e.g. Smooth-Particle Hydrodynamics (SPH) models by Revaz et al. (2009), Pasetto et al. (2011)), they mainly lack not only of a self-consistent treatment of various internal processes, but focussed on particular aspects only. Their results do not deviate too much from observational data, however, the system of satellites is exposed to a whole bunch of external processes also, like e.g. ram-pressure (Mayer et al. 2007) and tidal (Read et al. 2006) stripping, gas accretion, and further 

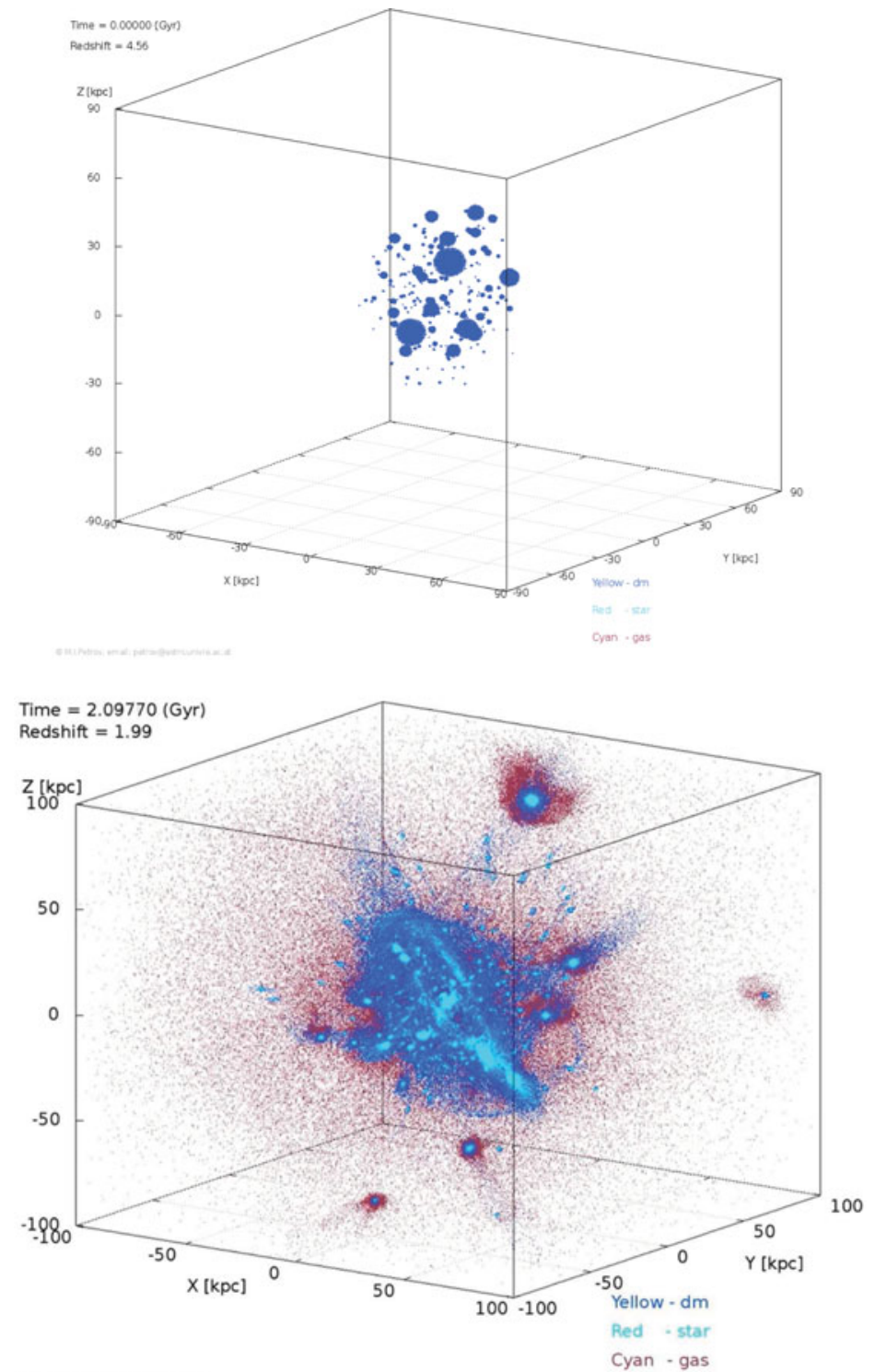

Figure 1. Cubes of $200 \mathrm{kpc}$ length around the Milky Way (at their center). upper panel: Initial conditions of the Milky Way's satellite system: Distribution of Dark Matter (DM) subhalos within a sphere of $40 \mathrm{kpc}$ radius around the Milky Way at redshift $\mathrm{z}=4.56$. lower panel: Snapshot of the satellites' dynamical evolution 2.1 Gyr after the numerical onset, i.e. at redshift $z=2$. The DM subhalos are filled with $17 \%$ baryonic gas mass, form stars, and lose mass of all constituents due to various effects (see text).

more. If these are not taken into account but simulations are limited to isolation DGs the models cannot allow a reliable trace-back of the evolution of any dSph galaxy.

In addition, simple non-dynamical considerations as performed to understand the chemical evolution (Lanfranchi et al. 2006, Lanfranchi \& Matteucci 2010, Prantzos 2008, Kirby et al. 2011a, Kirby et al. 2011b) provide only a limited understanding of the real evolution of dSphs. 
Font et al. (2006) investigated the nature of the progenitors of the stellar halo for a set of MWG-type galaxies and studied the chemical enrichment patterns in the context of the CDM model with a combination of semi-analytic prescriptions. They concluded that the difference in chemical abundance patterns in local halo stars versus surviving satellites arises naturally from the predictions of the hierarchical structure formation in a CDM universe.

Here we present a model of the early evolution and mass assembly history of the MWG's halo by the system of satellite galaxies treated in the gravitational field of the MWG. For this purpose we select a MWG-like DM host halo from the cosmological $\Lambda C D M$ simulation Via Lactea II (Diemand et al. 2008). The constraints are, that it does not undergo a major merger event over the Hubble time and that sufficient subhalos exist which allow the accretion by the host galaxy. For the simulations an advanced version of the single-gas chemo-dynamical SPH/N-body code is applied, treating the production and chemical evolution of 11 elements.

Since the acceptable computational time limits the number of gas particles to two million and the DM particles to the same order and because we aim at reaching a mass resolution of $10^{3} M_{\odot}$ per SPH particle, only 250 subhalos in the total-mass range of $10^{6}<M_{\text {sat }} / M_{\odot}<6 \times 10^{8}$ could be followed from redshift $z=4.56$ with its baryonic content. Unfortunately, this fact limits the radius of consideration to $40 \mathrm{kpc}$ around the MWG's center of mass. In order to study the construction of the MWG halo by accretion of subhalos with both gas and stars, as a first step, the chemo-dynamical evolution of the dSph system is followed for the first 2 Gyr, i.e. until redshift $z=2$ (see Fig.1).

Starting with a $10^{4} \mathrm{~K}$ warm gas of $17 \%$ of the subhalo masses in virial equilibrium and under the assumption that re-ionization is improbable to have affected the LG dSphs (Grebel \& Gallagher 2004), cooling allows the gas particles to achieve SF conditions in all satellites, but its efficiency directly depends on the mass of a satellite and its dynamical history (e.g. merging with other satellites or disruption by the MWG gravitational potential).

\section{The Milky Way halo formation}

Fig. 1 shows the evolution of the DM-gas-stars composit of the satellite galaxies. The SF starts in all satellites almost simultaneously, then ceases for the lowest mass objects, while it continues in more massive ones with fluctuations due to gas loss but also interactions with other objects. dSphs develop their stellar components and element abundances dependent on the distance from the MWG. Gas is pushed out from low-mass dSphs by their internal stellar energy release and lost from massive dSphs more by the tidal force. Both effects feed the MWG gas halo by pre-processed hot gas (fig. 2). Inherently as an additional effect, dSphs also get rid of their gas by their motion within the bath of their lost hot gas. Stars are also disrupted from the satellites and accumulate in the MWG halo at the early stages from all objects with low metallicity. Lateron, only stars from the massive satellites contribute due the cessation of SF in the less-massive systems. In total, $1.88 \times 10^{8} M_{\odot}$ of gas and $9.53 \times 10^{7} M_{\odot}$ of stellar mass are torn off from the satellite galaxies and got bound to the Galactic halo. From the same demolishing effect additionally $2.63 \times 10^{9} M_{\odot}$ of DM mass fed the MWG.

For the first 0.1 Gyrs of the simulation there is a considerable variance of stellar oxygen abundance in the whole system $(-5 . \leqslant[O / H] \leqslant-0.5)$ reflecting the very inhomogeneous production and distribution of enriched gas. After 0.1 Gyrs merging of the satellites' ISM promotes the mixing of heavy elements. Finally, almost complete recycling of the gas erases the abundance inhomogeneities so that oxygen in stars converges to 


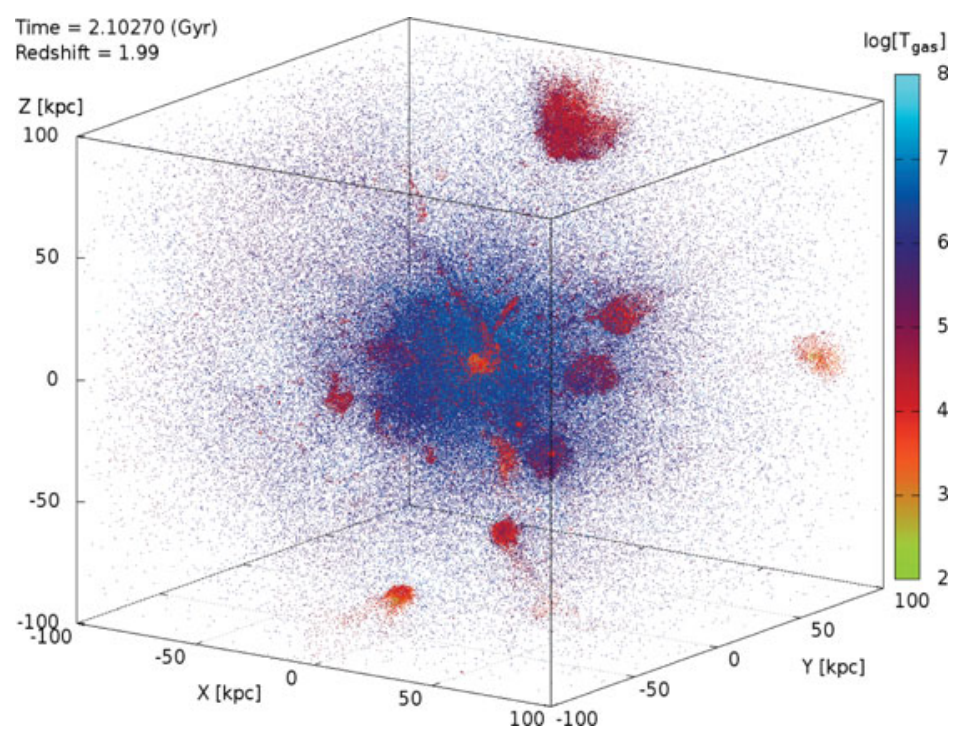

Figure 2. Snapshot of the gas distribution $2.1 \mathrm{Gyr}$ after the numerical onset, i.e. at redshift $\mathrm{z}=2.76$. The gas temperature is coloured according to the right colour panel.

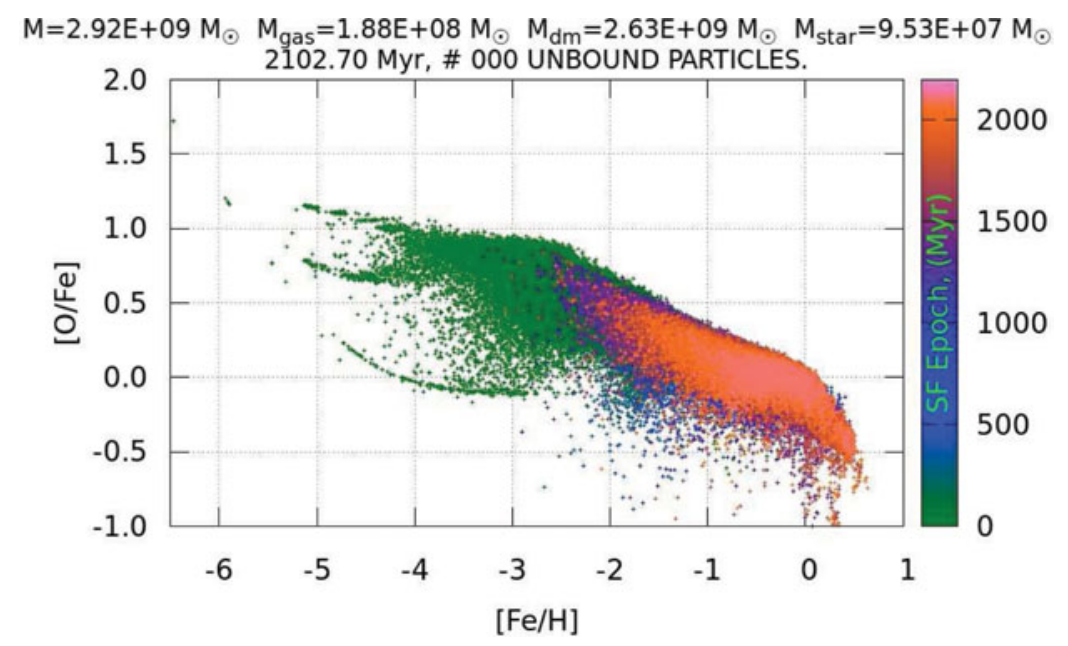

Figure 3. $[\mathrm{O} / \mathrm{Fe}]$ vs. $[\mathrm{Fe} / \mathrm{H}]$ distribution of star and gas that became unbound from the satellite galaxies within the first 2.1 Gyr after the model onset. The values of the element ratios are coloured according to the time when the stars and gas are dissolved from the satellites. The emerging stripes are artefacts by used values from tables during the first enrichment episode.

$-1 . \leqslant[\mathrm{Fe} / \mathrm{H}] \leqslant 0$. with a small dispersion (fig. 3). These high abundances show that the too efficient metal-enrichment in a single-gas phase treatment has to be relaxed by a more realistic chemo-dynamical multi-phase prescription of the ISM (Liu et al. 2015).

\section{Acknowledgements}

The authors are grateful to Simone Recchi for his contributions and continuous discussions on the chemical enrichment. This work was partly supported by the Austrian Science Foundation FWF under project no. P21097. 


\section{References}

Beers, T. \& Christlieb, N., 2005, Ann. Rev. A\&A, 43, 531

Bell, E.F., Zucker, D.B., Belokurov, V., et al., 2008, Astroph.J., 680, 295

Belokurov, V., Walker, M.G., Evans, N.W., et al., 2010, Astroph.J., 712, L103

Belokurov, V., Irwin, M.J., Koposov, S.E., et al., 2014, MNRAS, 441, 2124

Blumenthal, G.R., Primak, J.R., \& Rees, M.J., 1984, Nature, 311, 517

Carollo, D., Beers, T.C., Lee, Y.S., et al., 2007, Nature, 450, 1020

Diemand, J., Kuhlen, M., Madau, P., et al., 2008, Nature, 454, 735

Eggen, O.J., Lynden-Bell, D., \& Sandage, A.R., 1961, Astroph.J., 136, 748

Font, A.S., Johnston, K.V., Bullock, J.S., \& Robertson, B.E., 2006, Astroph.J., 638, 585

Frebel, A., Aoki, W., Christlieb, N., et al., 2005, Nature, 434, 871

Frebel, A., Kirby, E.N., \& Simon, J.D., 2010, Nature, 464, 72

Frebel, A., \& Bromm, V., 2012, Astroph.J., 759, 115

Frebel, A., \& Norris, J.E., 2015, Ann. Rev. A\&A, 53, 631

Grebel, E.K., \& Gallagher, J.S., 2004, Astroph.J., 610, L89

Hensler, G., Theis, C., \& Gallagher, J.S., 2004, Astron.Astroph., 426, 25

Ibata, R.A., Gilmore, G., \& Irwin, M.J., 1994, Nature,370, 194

Jerjen, H., Da Costa, G.S., Willman, B., et al., 2013, Astroph.J., 769, 14

Johnston, K.V., Bullock J.S., Sharma S., et al., 2008, Astroph.J., 689, 936

Kirby, E.N., Simon, J.D., Geha, M., et al., 2008, Astroph.J., 685, L43

Kirby, E.N., Lanfranchi, G.A., Simon, J.D., et al., 2011a, Astroph.J., 727, 78

Kirby, E.N., Cohen, J.G., Smith, G.H, et al., 2011b, Astroph.J., 727, 79

Koch, A., Grebel, E.K., Gilmore, G.F., et al., 2008, Astron.J., 135, 1580

Koch, A., 2009, Rev. Modern Astron., 21, 9

Lanfranchi, G.A., Matteucci, F., \& Cescutti, G., 2006, Astron.Astroph., 453, 67

Lanfranchi, G.A., \& Matteucci, F., 2010, Astron.Astroph., 512, A85

Liu, L., Petrov, M., Hensler, G., et al., 2015, MNRAS, submitted

Lynden-Bell, D., \& Lynden-Bell, R.M., 1995, MNRAS, 275, 429

Majewski, S.R., Skrutskie, M.F., Gomez-Flechoso, M.A., et al. 2013, Astroph.J., 599, 1082

Mayer, L., Kazantzidis, S., Mastropietro, C., \& Wadsley, J., 2007, Nature, 445, 738

Pasetto, S., Grebel, E.K., Berczik, P., \& Spurzem, R., 2011, Astron.Astroph., 525, A99

Prantzos, N., 2008, Astron.Astroph., 489, 525

Read, J.I., Wilkinson, M.I., Evans, N.W., et al., 2006, MNRAS, 366, 429

Revaz, Y., Jablonka P., Sawala T., et al., 2009, Astron.Astroph., 501, 189

Salvadori, S., Ferrara, A., \& Schneider, R., 2008, MNRAS, 386, 348

Searl, L., \& Zinn, R., 1978, Astroph.J., 225, 357

Shetrone, M., Venn, K.A., Tolstoy, E., et al., 2003, Astron.J., 125, 688

Springel, V., White, S.D.M., Jenkins, A., et al., 2005, Nature, 435, 629

Tolstoy, E., Venn, K.A., Shetrone, M., et al., 2003, Astron.J., 125, 707

Tolstoy, E., Hill, V., \& Tosi, M., 2009, Ann. Rev. A\& A, 47, 371

Venn, K.A., Irwin, M, Shetrone, M.D., et al., 2004. Astron.J., 128, 1177

White, S.D.M., \& Rees, M.J., 1978, MNRAS, 183, 341 